\title{
Double Resummation for Higgs Production
}

\author{
Marco Bonvini ${ }^{1, *}$ and Simone Marzani ${ }^{2, \dagger}$ \\ ${ }^{1}$ INFN, Sezione di Roma 1, Piazzale Aldo Moro 5, 00185 Roma, Italy \\ ${ }^{2}$ Dipartimento di Fisica, Università di Genova and INFN, Sezione di Genova, Via Dodecaneso 33, I-16146 Genova, Italy
}

(Received 26 February 2018; published 16 May 2018)

\begin{abstract}
We present the first double-resummed prediction of the inclusive cross section for the main Higgs production channel in proton-proton collisions, namely, gluon fusion. Our calculation incorporates to all orders in perturbation theory two distinct towers of logarithmic corrections which are enhanced, respectively, at threshold, i.e., large $x$, and in the high-energy limit, i.e., small $x$. Large- $x$ logarithms are resummed to next-to-next-to-next-to-leading logarithmic accuracy, while small- $x$ ones to leading logarithmic accuracy. The double-resummed cross section is furthermore matched to the state-of-the-art fixed-order prediction at next-to-next-to-next-to-leading accuracy. We find that double resummation corrects the Higgs production rate by $2 \%$ at the currently explored center-of-mass energy of $13 \mathrm{TeV}$ and its impact reaches $10 \%$ at future circular colliders at $100 \mathrm{TeV}$.
\end{abstract}

DOI: $10.1103 /$ PhysRevLett.120.202003

The major achievement of the first run of the CERN Large Hadron Collider (LHC) was the discovery of the Higgs boson [1,2], thus confirming the Brout-EnglertHiggs mechanism [3-6] for the electroweak symmetry breaking. The current and future runs of the LHC are rightly considered the Higgs precision era. The ATLAS and CMS Collaborations are continuously producing experimental analyses of ever increasing sophistication, which allow for a more detailed inspection of the Higgs sector. Examples include measurements of fiducial cross sections in different decay channels, as well as kinematic distributions of the Higgs boson [7-17]. In order to perform meaningful comparisons, it is imperative for the theoretical physics community to deliver calculations with uncertainties that match in magnitude those quoted by the experimental collaborations. Therefore, it does not come as a surprise that perturbative calculations in QCD have reached the astonishing next-to-next-to-next-to-leading order $\left(\mathrm{N}^{3} \mathrm{LO}\right)$ precision for the two most important Higgs production channels in proton-proton collisions: gluon fusion (GF), [18-25] and vector-boson fusion [26].

The calculation of the inclusive GF cross section is particularly challenging in QCD because, even at its lowest order, it proceeds via a massive quark loop [27]. Furthermore, this process is characterized by large perturbative QCD corrections. The NLO contribution (originally computed in Refs. [28,29] in an effective field theory (EFT)

Published by the American Physical Society under the terms of the Creative Commons Attribution 4.0 International license. Further distribution of this work must maintain attribution to the author(s) and the published article's title, journal citation, and DOI. Funded by SCOAP . approximation where the top-quark mass is considered much larger than any other scale, and in Ref. [30] for general quark mass running in the loop) is as large as the leading order, and the NNLO corrections (computed in Refs. [31-33] in the EFT and with finite top-mass corrections in Refs. [34-38]) are about half as large as the LO. The aforementioned EFT three-loop calculation of Refs. [18-22] finally shows perturbative convergence, with small theoretical uncertainties, as estimated by varying the arbitrary scales of the perturbative calculation.

A complementary approach to include higher-order corrections can be pursued using all-order techniques, which rely upon factorization properties of QCD matrix elements and phase space in particular kinematic limits. In the case of the inclusive GF cross section, one of the most studied and well-developed all-order techniques is threshold (or large $x$ ) resummation [39-41], which has been pushed to next-to-next-to-next-to-leading logarithmic $\left(\mathrm{N}^{3} \mathrm{LL}\right)$ accuracy $[18,42-48]$, and leads to a significantly improved convergence of the perturbative expansion [49]. In the opposite kinematic limit, i.e., at high energy (or small $x$ ), resummation [50-54] is also possible. In particular, small- $x$ resummation of splitting functions [55], which govern the evolution of the parton distribution functions (PDFs), was obtained in Refs. [56-65] (see also Refs. [6680]). Furthermore, high-energy resummation of partonic coefficient functions can be achieved in the framework of $k_{t}$ factorization $[81,82]$, and improved with the inclusion of subleading (but important) running coupling corrections [63]. All-order calculations in this framework have been exploited to assess and improve the aforementioned EFT, which is known to fail in the high-energy limit [83-85]. However, thus far, investigations in the high-energy regime were performed at fixed order, i.e., by expanding out the 
all-order results. All-order phenomenological results at small $x$ will be presented in this work for the first time.

In this Letter, we combine the above all-order approaches, together with the state-of-the-art fixed-order calculation, to obtain the most accurate prediction for Higgs production at the LHC. To our knowledge, it is the first time that double (large- and small- $x$ ) resummation is achieved. This breakthrough is possible because of two distinct advancements in the field. On the one hand, a general framework to combine the two resummations has been developed in Ref. [86] and implemented in public codes TROLL [45,49] and HELL $[87,88]$ so that numerical results can be easily obtained. On the other hand, recently, all-order calculations have been considered in the context of PDF determination, both at large- $x$ [89] and at small- $x[90,91]$. This opens up the possibility of achieving fully consistent resummed results. While we presently concentrate on the Higgs production cross section, our technique is fully general and can be applied to other important processes, such as the Drell-Yan process or heavy-quark production. We leave further phenomenological analyses to future work.

Let us start our discussion by introducing the factorized Higgs production cross section

$$
\begin{aligned}
\sigma\left(\tau, m_{H}^{2}\right)= & \tau \sigma_{0}\left(m_{H}^{2}, \alpha_{s}\left(\mu_{R}^{2}\right)\right) \\
& \times \sum_{i j} \int_{\tau}^{1} \frac{d x}{x} \mathcal{L}_{i j}\left(\frac{\tau}{x}, \mu_{F}^{2}\right) \\
& \times C_{i j}\left(x, \alpha_{s}\left(\mu_{R}^{2}\right), \frac{m_{H}^{2}}{\mu_{F}^{2}}, \frac{m_{H}^{2}}{\mu_{R}^{2}}\right),
\end{aligned}
$$

where $\sigma_{0}$ is the lowest-order partonic cross section, $\mathcal{L}_{i j}$ are parton luminosities (convolutions of PDFs), $C_{i j}$ are the perturbative partonic coefficient functions, $\tau=m_{H}^{2} / s$ is the squared ratio between the Higgs mass and the collider center-of-mass energy, and the sum runs over all parton flavors. Henceforth, we suppress the dependence on renormalization and factorization scales $\mu_{R}, \mu_{F}$. Moreover, because the Higgs boson couples to the gluon via a heavy-flavor loop, Eq. (1) also implicitly depends on any heavy virtual particle mass.

The general method to consistently combine large- and small- $x$ resummation of partonic coefficient functions $C_{i j}\left(x, \alpha_{s}\right)$ was developed in Ref. [86]. The basic principle is the definition of each resummation such that they do not interfere with each other. This statement can be made more precise by considering Mellin $(N)$ moments of Eq. (1). The key observation is that while in momentum $(x)$ space coefficient functions are distributions, their Mellin moments are analytic functions of the complex variable $N$ and therefore, they are (in principle) fully determined by the knowledge of their singularities. Thus, high-energy and threshold resummations are consistently combined if they mutually respect their singularity structure. In Ref. [86], where an approximate $\mathrm{N}^{3} \mathrm{LO}$ result for $C_{i j}$ was obtained by expanding both resummations to $\mathcal{O}\left(\alpha_{s}^{3}\right)$, the definition of the large- $x$ logarithms from threshold resummation was improved in order to satisfy the desired behavior, and later this improvement was extended to all orders in Ref. [45], leading to the socalled $\psi$-soft resummation scheme. Thanks to these developments, double-resummed partonic coefficient functions can be simply written as the sum of three terms [92]

$C_{i j}\left(x, \alpha_{s}\right)=C_{i j}^{\mathrm{fo}}\left(x, \alpha_{s}\right)+\Delta C_{i j}^{\mathrm{l} x}\left(x, \alpha_{s}\right)+\Delta C_{i j}^{\mathrm{s} x}\left(x, \alpha_{s}\right)$,

where the first term is the fixed-order calculation, the second one is the threshold-resummed $\psi$-soft contribution minus its expansion (to avoid double counting with the fixed-order), and the third one is the resummation of small- $x$ contributions, again minus its expansion. Note that not all partonic channels contribute to all terms in Eq. (2). For instance, the $q g$ contribution is power suppressed at threshold but it does exhibit logarithmic enhancement at small $x$.

Our result brings together the highest possible accuracy in all three contributions. The fixed-order piece is $\mathrm{N}^{3} \mathrm{LO}$ [18-22], supplemented with the correct small- $x$ behavior, as implemented in the public code ggHiggs $[49,86,93]$. Threshold-enhanced contributions are accounted for to next-to-next-to-next-to-leading logarithmic accuracy $\left(\mathrm{N}^{3} \mathrm{LL}\right)$ in the $\psi$-soft scheme, as implemented in the public code TROLL $[45,49]$. Finally, for high-energy resummation we consider the resummation of the leading nonvanishing tower of logarithms (here LLx) to the coefficient functions $[63,84]$, which we have now implemented in the code HELL $[87,88]$. The technical details of the implementation will be presented elsewhere [94]. Additionally, on top of scale variations, subleading terms can be varied in both resummed contributions, thus allowing for the estimate of the uncertainty from missing higher orders and from the matching procedure. Our calculation keeps finite top-mass effects where possible. In particular, in the fixed-order part they are included up to NNLO and in the thresholdresummed one up to NNLL. Furthermore, the small- $x$ contribution, both at fixed order and to all orders, must be computed with finite top mass, essentially because the limits $x \rightarrow 0$ and $m_{t} \rightarrow \infty$ do not commute. We will discuss further corrections associated with the masses of bottom and charm quarks when presenting our final results.

Having determined the resummation of the partonic coefficient functions, we now discuss the role of the parton luminosities $\mathcal{L}_{i j}$ that enter Eq. (1). Ideally, we would like to use PDFs that have been fitted using a double-resummed theory. However, this is clearly not possible. Indeed, this is the first study that aims to combine threshold and highenergy resummation, so a PDF fit with this theory will only appear in the future. Therefore, we have to find an acceptable compromise. Within the NNPDF framework [95], PDFs with threshold resummation were obtained in Ref. [89], while small- $x$ resummation was considered in Ref. [90]. We note that the inclusion of the latter was a 

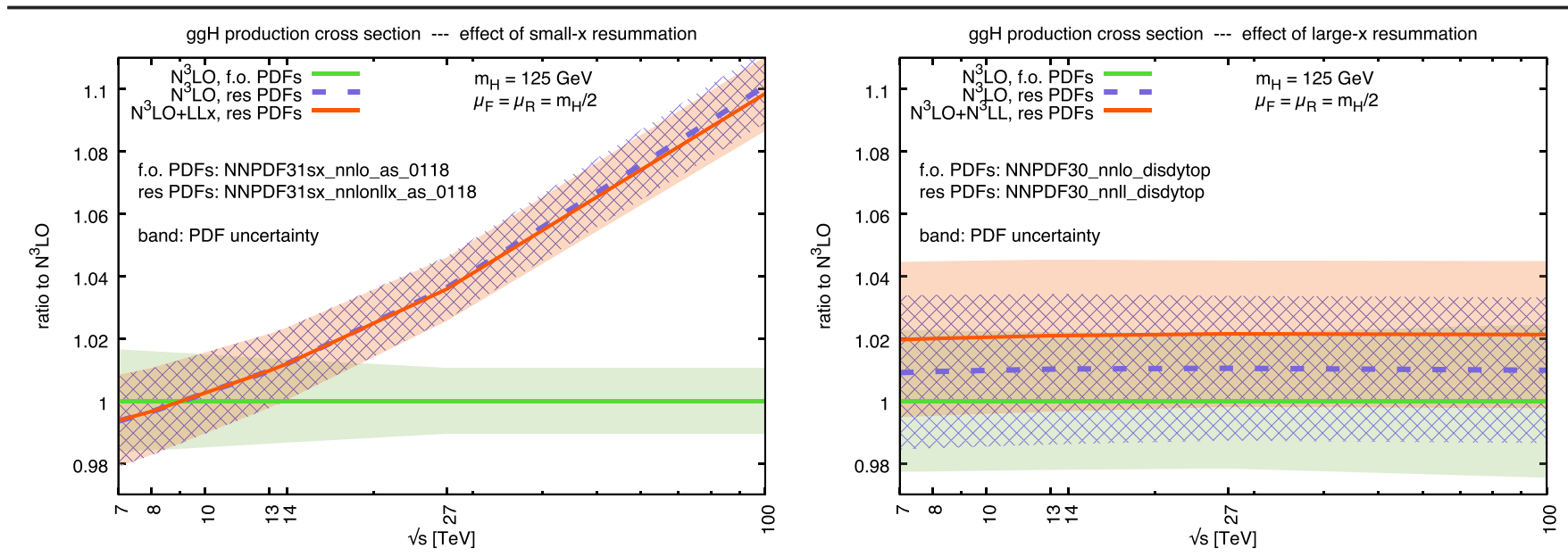

FIG. 1. All-order effects on the Higgs cross section computed at $\mathrm{N}^{3} \mathrm{LO}$, as a function of $\sqrt{s}$. The plot of the left shows the impact of small- $x$ resummation, while the one of the right of large- $x$ resummation. The bands represent PDF uncertainties.

challenging enterprise because small- $x$ logarithms appear both in coefficient functions and PDF evolution, while in the M S scheme large- $x$ resummation only affects coefficient functions $[96,97]$. In order to make an informed decision, we separately consider in Fig. 1 the impact of small- $x$ resummation (on the left) and large- $x$ resummation (on the right) on the GF cross section, as a function of the center-of-mass energy of the colliding protons.

Let us start by illustrating the situation concerning small$x$ resummation (left-hand plot). The plot shows the ratio of resummed results to the fixed order one, computed at $\mathrm{N}^{3} \mathrm{LO}$ with the fixed-order NNLO set of Ref. [90]. We include resummation in two steps. First (dashed blue), we compute the $\mathrm{N}^{3} \mathrm{LO}$ cross section using the "resummed PDFs" of Ref. [90], i.e., those fitted including resummation and evolving with NNLO + NLL $x$ theory. Then (solid red) we add the LL $x$ resummation to the Higgs coefficient functions, which provides the consistent resummed result. In all cases, the bands correspond to PDF uncertainties. The plot clearly shows that small- $x$ resummation has a modest effect at current LHC energies, but its impact grows substantially with the energy, reaching the $10 \%$ level at $100 \mathrm{TeV}$, heralding the fact that electroweak physics at $100 \mathrm{TeV}$ is small- $x$ physics. The plot also shows that the bulk of the effect comes from the resummed PDFs and their resummed evolution, while small- $x$ resummation of the Higgs coefficient functions is only a small correction. This perhaps surprising result can be understood by noting that, while the high-energy behavior of the PDFs is essentially determined by deep-inelastic scattering data at small $x$ and low $Q^{2}$, the Higgs cross section is characterized by a much higher value of $Q^{2}$, and it is dominated by soft emissions [98]. Furthermore, the large discrepancy between resummed and NNLO PDFs at large $\sqrt{s}$ is a manifestation of the perturbative instability of the latter. Indeed, as discussed at length in Ref. [90], resummed PDFs are close to the NLO ones, while the NNLO set significantly deviates at small $x$.
The situation is rather different if we analyze large- $x$ resummation (right-hand plot). Here we use the PDFs of Ref. [89], obtained with either NNLO and NNLO + NNLL theory, which, however, suffer from a larger uncertainty compared to standard global fits because of the reduced data set used in their determination. In this case the impact of the resummation on the $\mathrm{N}^{3} \mathrm{LO}$ cross section is smaller and fairly constant in the whole energy range considered here. The plot shows that about half of the $2 \%$ effect originates from the resummation in the PDFs (dashed blue), which is, however, not significant due to the large PDF uncertainties, and the other half by the resummation in the coefficient functions (solid red).

Therefore, by comparing the two plots in Fig. 1 we conclude that, lacking double-resummed PDFs, the use of small- $x$ resummed PDFs is preferred for the fairly large energy range considered here, because threshold-resummation effects in PDFs have a much smaller impact on the Higgs cross section. From the plots one may wonder whether double resummation of the coefficient functions is at all needed for phenomenology. Certainly its impact is numerically modest but we argue that its inclusion brings significant advantages both at small and large $x$. First, it allows for a fully consistent treatment at small $x$. Furthermore, the inclusion of large- $x$ resummation, although being a small correction to the $\mathrm{N}^{3} \mathrm{LO}$ results, allows for a more robust estimate of the theoretical uncertainty $[45,49]$.

We present double-resummed results for the Higgs cross section in GF in Fig. 2, where we show three plots at representative center-of-mass energies of the colliding protons. We consider the current energy of the LHC, $\sqrt{s}=13 \mathrm{TeV}$, and two possible energies for future colliders, namely, $\sqrt{s}=27 \mathrm{TeV}$ (HE-LHC) and $\sqrt{s}=100 \mathrm{TeV}$ (FCC). We choose as central scale $\mu_{F}=\mu_{R}=m_{H} / 2$. Numerical results are presented in Table I, where we also report for completeness the correction $\Delta \sigma_{b, c}$ to the fixedorder calculation due to the presence of massive bottom and 

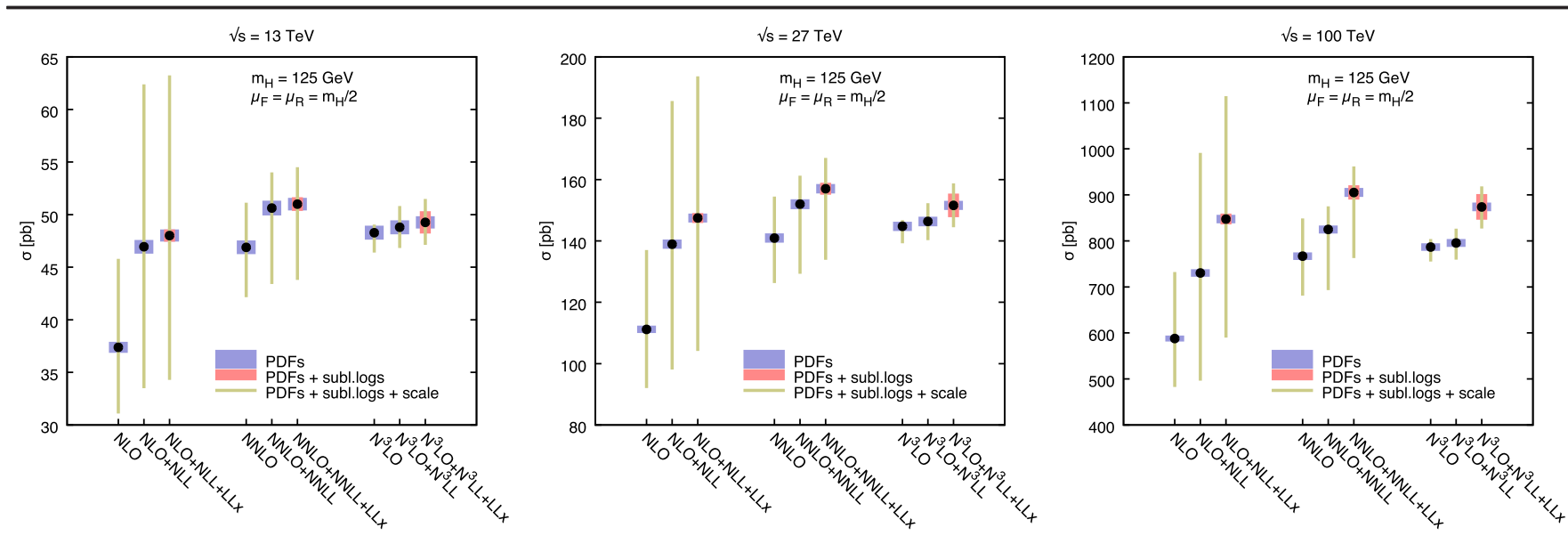

FIG. 2. Perturbative progression of the Higgs inclusive cross section in different approximations: fixed-order, threshold resummation, and double resummation, at three representative values of the collision energy.

charm quarks running in the loop, following the recommendation of Ref. [99]. Furthermore, electroweak corrections in the factorized approach, when included, amount to an extra $5 \%$ increase [99].

Each plot shows the perturbative progression of the cross section as obtained in different approximations: fixedorder, fixed-order and threshold, fixed-order and double resummation. We also show the three main contributions to the theoretical uncertainty, namely, PDFs, subleading logarithms at small- $x$ and scale variation. The latter also includes an estimate of subleading corrections at large $x$, resulting in 42 variations, as detailed in Ref. [49]. The uncertainty due to subleading logarithms at small $x$ has been determined by taking the envelope of two variants of the coefficient-function resummation, which take as input resummed splitting functions either at LL $x$ (to be precise, it is a modification of LL $x$ resummation which was called LL' in Refs. [87,88]) or at NLL $x$ [90,94]. We note that the PDFs are, in principle, affected by analogous uncertainty, which, however, is not currently included in their determination. Thus, the overall small- $x$ uncertainty might be underestimated. A qualitative assessment of this uncertainty was performed in Ref. [90] and its impact on the Higgs cross section will be investigated in Ref. [94].
We note that double resummation, mostly because of its threshold component, has a much more stable perturbative progression than its fixed-order counterpart: convergence is faster and uncertainty bands always cover the next perturbative order and shrink as higher orders are included [49]. While double resummation is a small $(2 \%)$ correction to the $\mathrm{N}^{3} \mathrm{LO}$ at current LHC energies, because of its small- $x$ component its impact grows with $\sqrt{s}$, becoming $5 \%$ at $27 \mathrm{TeV}$, before reaching approximately $10 \%$ at $100 \mathrm{TeV}$. Furthermore, we point out that a large contribution to the theoretical uncertainty originates from unknown subleading logarithms at small $x$. As a consequence, our doubleresummed prediction exhibits larger uncertainties than the $\mathrm{N}^{3} \mathrm{LO}$ one. On the one hand this highlights the importance of pushing the resummation of coefficient functions at small $x$ one order higher. On the other hand, this also implies that the uncertainty from missing higher orders is likely underestimated in a purely fixed-order approach, mostly due to the fact that PDF uncertainty does not fully account for it. Thus, even at LHC energies where its impact is modest, double resummation provides a more reliable estimate of the theoretical uncertainty affecting the Higgs cross section.

TABLE I. Values of the $\mathrm{N}^{3} \mathrm{LO}$ and $\mathrm{N}^{3} \mathrm{LO}+\mathrm{N}^{3} \mathrm{LL}+\mathrm{LL} x$ GF cross section for selected values of the collider energy and Higgs mass $m_{H}=125 \mathrm{GeV}$. We use the NNPDF31sx PDFs with $\alpha_{s}\left(m_{Z}^{2}\right)=0.118, m_{t}=173 \mathrm{GeV}, m_{b}=4.92 \mathrm{GeV}$, and $m_{c}=1.51 \mathrm{GeV}$.

\begin{tabular}{|c|c|c|c|c|c|c|c|c|}
\hline \multirow[b]{2}{*}{$\sqrt{s}$} & \multicolumn{3}{|c|}{ NNPDF31sx_nnlo_as_0118 } & \multicolumn{5}{|c|}{ NNPDF31sx_nnlonllx_as_0118 } \\
\hline & $\sigma_{\mathrm{N}^{3} \mathrm{LO}}$ & $\delta_{\text {scale }}$ & $\delta_{\mathrm{PDFs}}$ & $\sigma_{\mathrm{N}^{3} \mathrm{LO}} \mathrm{N}^{3} \mathrm{LL}+\mathrm{LL} x$ & $\delta_{\text {scale }}^{42 \mathrm{var}}$ & $\delta_{\mathrm{PDFs}}$ & $\delta_{\text {subl.logs }}$ & $\Delta \sigma_{b, c}$ \\
\hline $7 \mathrm{TeV}$ & $16.76 \mathrm{pb}$ & ${ }_{-3.7}^{+0.7} \%$ & $\pm 1.7 \%$ & $16.83 \mathrm{pb}$ & ${ }_{-3.6}^{+4.2} \%$ & $\pm 1.5 \%$ & $\pm 1.3 \%$ & $-1.01 \mathrm{pb}$ \\
\hline $8 \mathrm{TeV}$ & $21.32 \mathrm{pb}$ & ${ }_{-3.7}^{+0.7 \%}$ & $\pm 1.6 \%$ & $21.47 \mathrm{pb}$ & ${ }_{-3.6}^{+4.1} \%$ & $\pm 1.4 \%$ & $\pm 1.4 \%$ & $-1.26 \mathrm{pb}$ \\
\hline $13 \mathrm{TeV}$ & $48.28 \mathrm{pb}$ & ${ }_{-3.7}^{+0.9} \%$ & $\pm 1.4 \%$ & $49.26 \mathrm{pb}$ & ${ }_{-3.8}^{+4.0} \%$ & $\pm 1.2 \%$ & $\pm 1.8 \%$ & $-2.66 \mathrm{pb}$ \\
\hline $14 \mathrm{TeV}$ & $54.32 \mathrm{pb}$ & $\begin{array}{l}+0.9 \% \\
-3.7\end{array}$ & $\pm 1.3 \%$ & $55.56 \mathrm{pb}$ & $\begin{array}{l}+4.0 \% \\
-3.8\end{array}$ & $\pm 1.2 \%$ & $\pm 1.9 \%$ & $-2.96 \mathrm{pb}$ \\
\hline $27 \mathrm{TeV}$ & $144.7 \mathrm{pb}$ & ${ }_{-3.7}^{+0.9} \%$ & $\pm 1.1 \%$ & $151.6 \mathrm{pb}$ & ${ }_{-4.0}^{+4.0} \%$ & $\pm 1.0 \%$ & $\pm 2.3 \%$ & $-7.2 \mathrm{pb}$ \\
\hline $100 \mathrm{TeV}$ & $786.7 \mathrm{pb}$ & $\begin{array}{l}+1.9 \% \\
-3.8\end{array}$ & $\pm 1.1 \%$ & $873.9 \mathrm{pb}$ & $\begin{array}{l}+4.0 \% \\
-4.3 \%\end{array}$ & $\pm 1.2 \%$ & $\pm 3.0 \%$ & $-32.0 \mathrm{pb}$ \\
\hline
\end{tabular}


In this Letter we have presented, for the first time, results in perturbative QCD that supplement a fixed-order calculation with both threshold and high-energy resummation. We have applied our double-resummed framework to calculate the inclusive cross section for Higgs production in gluon fusion. Our result features the state-of-the art accuracy $\mathrm{N}^{3} \mathrm{LO}+\mathrm{N}^{3} \mathrm{LL}+\mathrm{LL} x$ and crucially, it makes use of recently determined resummed parton distributions. The method presented here is rather general and it can be applied to a variety of processes currently studied at the LHC, such as electroweak-boson production or top-quark production. Furthermore, we anticipate that its generalization to differential distributions, such as rapidity and transverse momentum, is possible and we look forward to future work in this direction.

We thank R. D. Ball, S. Forte, G. Ridolfi, and L. Rottoli for many useful discussions. The work of M. B. is supported by the Marie Skłodowska-Curie Grant HiPPiE@LHC.

*marco.bonvini@roma1.infn.it †simone.marzani@ge.infn.it

[1] G. Aad et al. (ATLAS Collaboration), Observation of a new particle in the search for the Standard Model Higgs boson with the ATLAS detector at the LHC, Phys. Lett. B 716, 1 (2012).

[2] S. Chatrchyan et al. (CMS Collaboration), Observation of a new boson at a mass of $125 \mathrm{GeV}$ with the CMS experiment at the LHC, Phys. Lett. B 716, 30 (2012).

[3] F. Englert and R. Brout, Broken Symmetry and the Mass of Gauge Vector Mesons, Phys. Rev. Lett. 13, 321 (1964).

[4] P. W. Higgs, Broken symmetries, massless particles and gauge fields, Phys. Lett. 12, 132 (1964).

[5] P. W. Higgs, Broken Symmetries and the Masses of Gauge Bosons, Phys. Rev. Lett. 13, 508 (1964).

[6] P. W. Higgs, Spontaneous symmetry breakdown without massless bosons, Phys. Rev. 145, 1156 (1966).

[7] M. Aaboud et al. (ATLAS Collaboration), Measurement of inclusive and differential cross sections in the $H \rightarrow Z Z^{*} \rightarrow$ $4 \ell$ decay channel in $p p$ collisions at $\sqrt{s}=13 \mathrm{TeV}$ with the ATLAS detector, J. High Energy Phys. 10 (2017) 132.

[8] M. Aaboud et al. (ATLAS Collaboration), Measurement of the Higgs boson coupling properties in the $H \rightarrow Z Z^{*} \rightarrow 4 \ell$ decay channel at $\sqrt{s}=13 \mathrm{TeV}$ with the ATLAS detector, J. High Energy Phys. 03 (2018) 095.

[9] G. Aad et al. (ATLAS, CMS Collaborations), Measurements of the Higgs boson production and decay rates and constraints on its couplings from a combined ATLAS and CMS analysis of the LHC pp collision data at $\sqrt{s}=7$ and 8 TeV, J. High Energy Phys. 08 (2016) 045.

[10] G. Aad et al. (ATLAS Collaboration), Measurement of fiducial differential cross sections of gluon-fusion production of Higgs bosons decaying to $W W^{*} \rightarrow e \nu \mu \nu$ with the ATLAS detector at $\sqrt{s}=8 \mathrm{TeV}$, J. High Energy Phys. 08 (2016) 104.
[11] G. Aad et al. (ATLAS Collaboration), Measurements of the Higgs boson production and decay rates and coupling strengths using pp collision data at $\sqrt{s}=7$ and $8 \mathrm{TeV}$ in the ATLAS experiment, Eur. Phys. J. C 76, 6 (2016).

[12] G. Aad et al. (ATLAS Collaboration), Measurements of the Total and Differential Higgs Boson Production Cross Sections Combining the $H \rightarrow \gamma \gamma$ and $H \rightarrow Z Z^{*} \rightarrow 4 l$ Decay Channels at $\sqrt{s}=8 \mathrm{TeV}$ with the ATLAS Detector, Phys. Rev. Lett. 115, 091801 (2015).

[13] A. M. Sirunyan et al. (CMS Collaboration), Measurement of the $\mathrm{Z}^{*} \rightarrow \tau \tau$ cross section in pp collisions at $\sqrt{s}=13 \mathrm{TeV}$ and validation of $\tau$ lepton analysis techniques, arXiv: 1801.03535

[14] A. M. Sirunyan et al. (CMS Collaboration), Measurements of properties of the Higgs boson decaying into the fourlepton final state in pp collisions at $\sqrt{s}=13 \mathrm{TeV}$, J. High Energy Phys. 11 (2017) 047.

[15] V. Khachatryan et al. (CMS Collaboration), Measurement of the transverse momentum spectrum of the Higgs boson produced in pp collisions at $\sqrt{s}=8 \mathrm{TeV}$ using $H \rightarrow W W$ decays, J. High Energy Phys. 03 (2017) 032.

[16] V. Khachatryan et al. (CMS Collaboration), Measurement of differential and integrated fiducial cross sections for Higgs boson production in the four-lepton decay channel in pp collisions at $\sqrt{s}=7$ and $8 \mathrm{TeV}$, J. High Energy Phys. 04 (2016) 005.

[17] V. Khachatryan et al. (CMS Collaboration), Measurement of differential cross sections for Higgs boson production in the diphoton decay channel in pp collisions at $\sqrt{s}=8 \mathrm{TeV}$, Eur. Phys. J. C 76, 13 (2016).

[18] C. Anastasiou, C. Duhr, F. Dulat, E. Furlan, T. Gehrmann, F. Herzog, and B. Mistlberger, Higgs boson gluon fusion production at threshold in $\mathrm{N}^{3} \mathrm{LO}$ QCD, Phys. Lett. B 737, 325 (2014).

[19] C. Anastasiou, C. Duhr, F. Dulat, E. Furlan, T. Gehrmann, F. Herzog, and B. Mistlberger, Higgs boson gluon fusion production beyond threshold in $\mathrm{N}^{3} \mathrm{LO}$ QCD, J. High Energy Phys. 03 (2015) 091.

[20] C. Anastasiou, C. Duhr, F. Dulat, F. Herzog, and B. Mistlberger, Higgs Boson Gluon-Fusion Production in QCD at Three Loops, Phys. Rev. Lett. 114, 212001 (2015).

[21] C. Anastasiou, C. Duhr, F. Dulat, E. Furlan, T. Gehrmann, F. Herzog, A. Lazopoulos, and B. Mistlberger, High precision determination of the gluon fusion Higgs boson cross-section at the LHC, J. High Energy Phys. 05 (2016) 058.

[22] B. Mistlberger, Higgs boson production at hadron colliders at $\mathrm{N}^{3} \mathrm{LO}$ in QCD, arXiv:1802.00833.

[23] Y. Li, A. von Manteuffel, R. M. Schabinger, and H. X. Zhu, Soft-virtual corrections to Higgs production at $\mathrm{N}^{3} \mathrm{LO}$, Phys. Rev. D 91, 036008 (2015).

[24] Y. Li, A. von Manteuffel, R. M. Schabinger, and H. X. Zhu, $\mathrm{N}^{3}$ LO Higgs boson and Drell-Yan production at threshold: The one-loop two-emission contribution, Phys. Rev. D 90, 053006 (2014).

[25] C. Anzai, A. Hasselhuhn, M. Höschele, J. Hoff, W. Kilgore, M. Steinhauser, and T. Ueda, Exact $\mathrm{N}^{3} \mathrm{LO}$ results for $q \bar{q} \rightarrow H+X$, J. High Energy Phys. 07 (2015) 140.

[26] F. A. Dreyer and A. Karlberg, Vector-Boson Fusion Higgs Production at Three Loops in QCD, Phys. Rev. Lett. 117, 072001 (2016). 
[27] H. M. Georgi, S. L. Glashow, M. E. Machacek, and D. V. Nanopoulos, Higgs Bosons from Two Gluon Annihilation in Proton Proton Collisions, Phys. Rev. Lett. 40, 692 (1978).

[28] S. Dawson, Radiative corrections to Higgs boson production, Nucl. Phys. B359, 283 (1991).

[29] A. Djouadi, M. Spira, and P. M. Zerwas, Production of Higgs bosons in proton colliders: QCD corrections, Phys. Lett. B 264, 440 (1991).

[30] M. Spira, A. Djouadi, D. Graudenz, and P. M. Zerwas, Higgs boson production at the LHC, Nucl. Phys. B453, 17 (1995).

[31] R. V. Harlander and W. B. Kilgore, Next-to-Next-to-Leading Order Higgs Production at Hadron Colliders, Phys. Rev. Lett. 88, 201801 (2002).

[32] C. Anastasiou and K. Melnikov, Higgs boson production at hadron colliders in NNLO QCD, Nucl. Phys. B646, 220 (2002).

[33] V. Ravindran, J. Smith, and W. L. van Neerven, NNLO corrections to the total cross-section for Higgs boson production in hadron-hadron collisions, Nucl. Phys. B665, 325 (2003).

[34] R. V. Harlander and K. J. Ozeren, Top mass effects in Higgs production at next-to-next-to-leading order QCD: Virtual corrections, Phys. Lett. B 679, 467 (2009).

[35] R. V. Harlander and K. J. Ozeren, Finite top mass effects for hadronic Higgs production at next-to-next-to-leading order, J. High Energy Phys. 11 (2009) 088.

[36] R. V. Harlander, H. Mantler, S. Marzani, and K. J. Ozeren, Higgs production in gluon fusion at next-to-next-to-leading order QCD for finite top mass, Eur. Phys. J. C 66, 359 (2010).

[37] A. Pak, M. Rogal, and M. Steinhauser, Virtual three-loop corrections to Higgs boson production in gluon fusion for finite top quark mass, Phys. Lett. B 679, 473 (2009).

[38] A. Pak, M. Rogal, and M. Steinhauser, Finite top quark mass effects in NNLO Higgs boson production at LHC, J. High Energy Phys. 02 (2010) 025.

[39] S. Catani, M. L. Mangano, P. Nason, and L. Trentadue, The resummation of soft gluon in hadronic collisions, Nucl. Phys. B478, 273 (1996).

[40] D. de Florian, M. Grazzini, and M. Grazzini, Higgs production at the LHC: updated cross sections at $\sqrt{s}=$ 8 TeV, Phys. Lett. B 718, 117 (2012).

[41] V. Ahrens, T. Becher, M. Neubert, and L. Lin Yang, Renormalization-group improved prediction for Higgs production at hadron colliders, Eur. Phys. J. C 62, 333 (2009).

[42] S. Moch, J. A. M. Vermaseren, and A. Vogt, Higher-order corrections in threshold resummation, Nucl. Phys. B726, 317 (2005).

[43] S. Moch and A. Vogt, Higher-order soft corrections to lepton pair and Higgs boson production, Phys. Lett. B 631, 48 (2005).

[44] E. Laenen and L. Magnea, Threshold resummation for electroweak annihilation from DIS data, Phys. Lett. B 632, 270 (2006).

[45] M. Bonvini and S. Marzani, Resummed Higgs cross section at $\mathrm{N}^{3}$ LL, J. High Energy Phys. 09 (2014) 007.

[46] S. Catani, L. Cieri, D. de Florian, G. Ferrera, and M. Grazzini, Threshold resummation at $\mathrm{N}^{3} \mathrm{LL}$ accuracy and soft-virtual cross sections at $\mathrm{N}^{3} \mathrm{LO}$, Nucl. Phys. B888, 75 (2014).
[47] M. Bonvini and L. Rottoli, Three loop soft function for $\mathrm{N}^{3} \mathrm{LL}^{\prime}$ gluon fusion Higgs production in soft-collinear effective theory, Phys. Rev. D 91, 051301 (2015).

[48] T. Schmidt and M. Spira, Higgs boson production via gluon fusion: Soft-gluon resummation including mass effects, Phys. Rev. D 93, 014022 (2016).

[49] M. Bonvini, S. Marzani, C. Muselli, and L. Rottoli, On the Higgs cross section at $\mathrm{N}^{3} \mathrm{LO}+\mathrm{N}^{3} \mathrm{LL}$ and its uncertainty, J. High Energy Phys. 08 (2016) 105.

[50] V. S. Fadin, E. A. Kuraev, and L. N. Lipatov, On the Pomeranchuk singularity in asymptotically free theories, Phys. Lett. 60B, 50 (1975).

[51] L. N. Lipatov, Reggeization of the vector meson and the vacuum singularity in nonabelian gauge theories, Sov. J. Nucl. Phys. 23, 338 (1976).

[52] E. A. Kuraev, L. N. Lipatov, and Victor S. Fadin, MultiReggeon processes in the Yang-Mills theory, Sov. Phys. JETP 44, 443 (1976).

[53] E. A. Kuraev, L. N. Lipatov, and V. S. Fadin, The Pomeranchuk singularity in nonabelian gauge theories, Sov. Phys. JETP 45, 199 (1977).

[54] I. I. Balitsky and L. N. Lipatov, The Pomeranchuk singularity in quantum chromodynamics, Sov. J. Nucl. Phys. 28, 822 (1978).

[55] S. Catani and F. Hautmann, Quark anomalous dimensions at small x, Phys. Lett. B 315, 157 (1993).

[56] R. D. Ball and S. Forte, Asymptotically free partons at highenergy, Phys. Lett. B 405, 317 (1997).

[57] R. D. Ball and S. Forte, The Small x behavior of AltarelliParisi splitting functions, Phys. Lett. B 465, 271 (1999).

[58] G. Altarelli, R. D. Ball, and S. Forte, Resummation of singlet parton evolution at small x, Nucl. Phys. B575, 313 (2000).

[59] G. Altarelli, R. D. Ball, and S. Forte, Small x resummation and HERA structure function data, Nucl. Phys. B599, 383 (2001).

[60] G. Altarelli, R. D. Ball, and S. Forte, Factorization and resummation of small $\mathrm{x}$ scaling violations with running coupling, Nucl. Phys. B621, 359 (2002).

[61] G. Altarelli, R. D. Ball, and S. Forte, An anomalous dimension for small x evolution, Nucl. Phys. B674, 459 (2003).

[62] G. Altarelli, R. D. Ball, and S. Forte, Perturbatively stable resummed small x evolution kernels, Nucl. Phys. B742, 1 (2006).

[63] R. D. Ball, Resummation of hadroproduction cross-sections at high energy, Nucl. Phys. B796, 137 (2008).

[64] G. Altarelli, R. D. Ball, and S. Forte, Structure function resummation in small-x QCD: Applications of quantum field theory to phenomenology, Proc. Sci. RADCOR2007 (2007) 028.

[65] G. Altarelli, R. D. Ball, and S. Forte, Small x resummation with quarks: Deep-inelastic scattering, Nucl. Phys. B799, 199 (2008).

[66] G. P. Salam, A resummation of large subleading corrections at small x, J. High Energy Phys. 07 (1998) 019.

[67] M. Ciafaloni and D. Colferai, The BFKL equation at next-to-leading level and beyond, Phys. Lett. B 452, 372 (1999).

[68] M. Ciafaloni, D. Colferai, and G. P. Salam, Renormalization group improved small x equation, Phys. Rev. D 60, 114036 (1999). 
[69] M. Ciafaloni, D. Colferai, and G. P. Salam, A collinear model for small x physics, J. High Energy Phys. 10 (1999) 017.

[70] M. Ciafaloni, D. Colferai, and G. P. Salam, On factorization at small x, J. High Energy Phys. 07 (2000) 054.

[71] M. Ciafaloni, D. Colferai, G. P. Salam, and A. M. Stasto, Expanding running coupling effects in the hard pomeron, Phys. Rev. D 66, 054014 (2002).

[72] M. Ciafaloni, D. Colferai, G. P. Salam, and A. M. Stasto, Renormalization group improved small x Green's function, Phys. Rev. D 68, 114003 (2003).

[73] M. Ciafaloni, D. Colferai, G. P. Salam, and A. M. Stasto, The Gluon splitting function at moderately small x, Phys. Lett. B 587, 87 (2004).

[74] M. Ciafaloni and D. Colferai, Dimensional regularisation and factorisation schemes in the BFKL equation at subleading level, J. High Energy Phys. 09 (2005) 069.

[75] M. Ciafaloni, D. Colferai, G. P. Salam, and A. M. Stasto, Minimal subtraction vs. physical factorisation schemes in small-x QCD, Phys. Lett. B 635, 320 (2006).

[76] M. Ciafaloni, D. Colferai, G. P. Salam, and A. M. Stasto, A matrix formulation for small- $x$ singlet evolution, J. High Energy Phys. 08 (2007) 046.

[77] R. S. Thorne, Explicit calculation of the running coupling BFKL anomalous dimension, Phys. Lett. B 474, 372 (2000).

[78] R. S. Thorne, NLO BFKL equation, running coupling and renormalization scales, Phys. Rev. D 60, 054031 (1999).

[79] R.S. Thorne, The Running coupling BFKL anomalous dimensions and splitting functions, Phys. Rev. D 64, 074005 (2001).

[80] C. D. White and R. S. Thorne, A global fit to scattering data with NLL BFKL resummations, Phys. Rev. D 75, 034005 (2007).

[81] S. Catani, M. Ciafaloni, and F. Hautmann, High energy factorization and small- $x$ heavy flavour production, Nucl. Phys. B366, 135 (1991).

[82] S. Catani and F. Hautmann, High-energy factorization and small x deep inelastic scattering beyond leading order, Nucl. Phys. B427, 475 (1994).

[83] F. Hautmann, Heavy top limit and double logarithmic contributions to Higgs production at $m_{H}^{2} / s$ much less than 1, Phys. Lett. B 535, 159 (2002).

[84] S. Marzani, R. D. Ball, V. Del Duca, S. Forte, and A. Vicini, Higgs production via gluon-gluon fusion with finite top mass beyond next-to-leading order, Nucl. Phys. B800, 127 (2008).
[85] R. S. Pasechnik, O. V. Teryaev, and A. Szczurek, Scalar Higgs boson production in a fusion of two off-shell gluons, Eur. Phys. J. C 47, 429 (2006).

[86] R. D. Ball, M. Bonvini, S. Forte, S. Marzani, and G. Ridolfi, Higgs production in gluon fusion beyond NNLO, Nucl. Phys. B874, 746 (2013).

[87] M. Bonvini, S. Marzani, and T. Peraro, Small- $x$ resummation from HELL, Eur. Phys. J. C 76, 597 (2016).

[88] M. Bonvini, S. Marzani, and C. Muselli, Towards parton distribution functions with small- $x$ resummation: HELL 2.0, J. High Energy Phys. 12 (2017) 117.

[89] M. Bonvini, S. Marzani, J. Rojo, L. Rottoli, M. Ubiali, R. D. Ball, V. Bertone, S. Carrazza, and N. P. Hartland, Parton distributions with threshold resummation, J. High Energy Phys. 09 (2015) 191.

[90] R. D. Ball, V. Bertone, M. Bonvini, S. Marzani, J. Rojo, and L. Rottoli, Parton distributions with small-x resummation: evidence for BFKL dynamics in HERA data, arXiv: 1710.05935 .

[91] H. Abdolmaleki et al. (xFitter Developers' Team), Impact of low- $x$ resummation on QCD analysis of HERA data, arXiv:1802.00064.

[92] The fixed-order and small- $x$ contributions are written in this form, while the large- $x$ one is written in $N$ space and its Mellin inversion is performed together with the parton luminosity using the so-called minimal prescription [39].

[93] M. Bonvini, R. D. Ball, S. Forte, S. Marzani, and G. Ridolfi, Updated Higgs cross section at approximate $\mathrm{N}^{3} \mathrm{LO}$, J. Phys. G 41, 095002 (2014).

[94] M. Bonvini, Small-x phenomenology at the LHC and beyond: HELL 3.0 and the case of the Higgs cross section (to be published).

[95] R. D. Ball et al. (NNPDF Collaboration), Parton distributions from high-precision collider data, Eur. Phys. J. C 77, 663 (2017).

[96] G. P. Korchemsky, Asymptotics of the Altarelli-ParisiLipatov evolution kernels of parton distributions, Mod. Phys. Lett. A 04, 1257 (1989).

[97] S. Albino and R. D. Ball, Soft resummation of quark anomalous dimensions and coefficient functions in MSbar factorization, Phys. Lett. B 513, 93 (2001).

[98] M. Bonvini, S. Forte, and G. Ridolfi, The Threshold Region for Higgs Production in Gluon Fusion, Phys. Rev. Lett. 109, 102002 (2012).

[99] D. de Florian et al. (LHC Higgs Cross Section Working Group), Handbook of LHC Higgs Cross Sections: 4. Deciphering the Nature of the Higgs Sector, arXiv:1610.07922. 